\title{
ArchiveWeb: Collaboratively Extending and Exploring Web Archive Collections
}

\author{
How would you like to work with your collections?
}

\author{
Zeon Trevor Fernando · Ivana Marenzi · Wolfgang Nejdl
}

Received: date / Accepted: date

\begin{abstract}
Curated web archive collections contain focused digital content which is collected by archiving organizations, groups, and individuals to provide a representative sample covering specific topics and events to preserve them for future exploration and analysis. In this paper, we discuss how to best support collaborative construction and exploration of these collections through the ArchiveWeb system. ArchiveWeb has been developed using an iterative evaluation-driven designbased research approach, with considerable user feedback at all stages.

The first part of this paper describes the important insights we gained from our initial requirements engineering phase during the first year of the project and the main functionalities of the current ArchiveWeb system for searching, constructing, exploring, and discussing web archive collections. The second part summarizes the feedback we received on this version from archiving organizations and libraries, as well as our corresponding plans for improving and extending the system for the next release.
\end{abstract}

Keywords Working with web archives, Collaborative search and exploration, Web archive requirements and evaluation

\section{Introduction}

The web is becoming an important corpus for studying human society by researchers in the humanities, social sciences, and computer sciences alike. Web archives collect, preserve, and provide ongoing access to ephemeral

Zeon Trevor Fernando · Ivana Marenzi · Wolfgang Nejdl

E-mail: \{fernando,marenzi,nejdl\}@L3S.de

L3S Research Center, Hannover, Germany web pages and hence encode important traces of human thought, activity, and history. Curated web archive collections contain focused digital content from archiving organizations, groups, and individuals related to specific topics or covering specific events, which are collected to provide representative samples and preserve them for future exploration and analysis. However, there have been only a few concerted efforts to provide tools and platforms for exploring and working with such web archives.

This article focuses on the ArchiveWeb ${ }^{1}$ platform which provides facilities to collaboratively explore and work with web collections in an interactive and userfriendly way, both for research and for learning. In particular we focus on supporting collaborative exploration and analytics through a user friendly searching and sharing interface (ArchiveWeb). Our goal is to allow users (e.g., archivists and librarians, web archive curators, researchers) to pose queries in the context of a larger exploration process where search results are stored for sharing with colleagues and collaborators, later discussion, and analysis [13]. This enables the user to consider dependencies between queries independent of the order in which they are posed, thereby regarding them in the context of later queries and query results. Building on previous work we did for collaborative learning environments [13], we enable users to build their collections incrementally and collaboratively, which is an important step to support their research work. We also allow researchers and their collaborators to approve, add, or remove results to their collections and to discuss their significance.

\footnotetext{
1 http://archiveweb.13s. uni-hannover.de/aw/index. jsf
} 
In the following section, we provide a short overview of related work, in Sect. 3, we describe a life cycle model for web archiving, and in Sect. 4, we give more details about the analysis of user requirements on which we based the ArchiveWeb interface. In Sect. 5, we describe two sample web archive collections we are working with. Section 6 includes a description of the main functionalities of our system, and Sect. 7 presents the evaluation design with a detailed discussion of the evaluation results. In Sect. 8, we summarize future extensions for the next ArchiveWeb release and finally draw conclusions in Sect. 9.

\section{Related Work}

Information retrieval (IR) models and algorithms have been extremely successful during the last 20 years in providing everybody with easy access to the vast amount of information available on and through the web. Web and IR conferences connect a large number of researchers working on problems related to web search, the Social Web and data mining, and related topics. Surprisingly little work has been spent, however, on issues related to temporal retrieval, or exploration and analysis of large temporal collections like web archives [4], even though the need for focusing research on these issues has been recognized $[1,18]$.

The Alexandria project ${ }^{2}$ focuses on open questions where radical changes and advances in the state of the art are necessary to move ahead in our abilities of indexing, retrieving, and exploring past web content. Models and algorithms for temporal information retrieval developed in the context of this project will take the unique temporal dimension of web archives into account. Semantic entity-based indexing will support exploration of temporal web content and evolving entities in a more user-oriented way than conventional document-based retrieval. Finally, user input gathered by complex and collaborative search and analysis processes of archive users will further enable us to improve web archive indexing and enrichment considerably. While this project targets web archives, similar issues arise in any digital archive, and solutions developed within Alexandria will likely be applicable to other types of archives as well.

Often desired search functionalities in web archives include full-text search with good ranking, followed by URL search [16]. A recent survey showed that $89 \%$ of web archives provide URL search access, and $79 \%$ give metadata search functionalities [7]. Some existing

\footnotetext{
2 http://alexandria-project.eu/
}

projects that provide limited support for web archive research are discussed below.

The Wayback Machine ${ }^{3}$ is a web archive access tool supported by the Internet Archive. It provides the ability to retrieve and access web pages stored in a web archive through URL search. The results for each URL are displayed in a calendar view which displays the number of times the URL was crawled by the Internet Archive web crawlers. Archive-It and ArchiveTheNet ${ }^{4}$ are web archive services provided by the Internet Archive and the Internet Memory Foundation. These services enable focused archiving of web contents by organizations, such as universities or libraries, that otherwise could not manage their own archives. The Memento Project ${ }^{5}$ enables the discovery of archived content from across multiple web archives via URL search.

A few researchers have worked on providing new interfaces and visualizations for searching, exploring, and discovering insights from web archives. Odijk et al. [14] present an exploratory search interface to improve accessibility of digital archived collections for humanities scholars, in order to highlight different perspectives across heterogeneous historical collections. The motivation for this work derives from the huge amount of digital material that has become available to study our recent history, including books, newspapers, and web pages, all of which provide different perspectives on people, places, and events over time. The authors connect heterogeneous digital collections through the temporal references found in the documents as well as their textual content, in order to support scholars to detect, visualize, and explore materials from different perspectives. Padia et al. [15] provided an overview of a web archive collection by highlighting the collection's underlying characteristics using different visualizations of image plots, wordle, bubble charts, and timelines. Lin et al. [10] present an interactive visualization based on topic models for exploring archived content, so that users can get an overview of the collection content. The visualization displays a person-by-topic matrix that shows the association between US senators' websites and the derived topics. The interface also provides drill-down capabilities for users to examine the pages in which a topic is prevalent.

All of the above tools and interfaces help support the exploration and search of web archives for individual users and researchers. In addition, ArchiveWeb aims at supporting the collaborative exploration of web archives. Previous research on helping users keep

\footnotetext{
3 http://archive.org/web/

4 http://archivethe.net/

5 http://timetravel.mementoweb.org
} 
track of their resources includes tools that provide better search and organizational facilities based on metadata/time [5] or tagging [3]. Our system provides similar organizational functionalities refined through close collaboration with several learning communities and previous work on the LearnWeb platform [13], thus gaining advantage from several years of development and user feedback in that context. ArchiveWeb builds on the LearnWeb experience which already supports collaborative sensemaking by allowing users to share and collaboratively work on resources retrieved from various web sources.

LearnWeb $^{6}$ is a learning and competence development environment, which allows users to share and collaboratively work on resources collected from the web or user-generated [13]. It provides users with a search interface for resource discovery and sharing across various Web 2.0 services such as YouTube, Flickr, and Slideshare, including LearnWeb itself, and offers a Personal Web 2.0 Learning Space. In order to support collaborative searching, LearnWeb provides automatic resource annotation. Resources in LearnWeb can be bookmarked, tagged, rated, and discussed by all users who are allowed to access them. Comments on particular learning resources can be used to enrich the description. Users can create folders to bundle resources that belong to the same learning context. Hence, the LearnWeb community can collaboratively identify the best learning resources for specific learning domains. A discussion of the full potentialities and affordances of LearnWeb as a collaborative platform is beyond the scope of this paper, but can be found in a series of published studies [11, $12,13]$.

The Integrated Digital Event Archive and Library (IDEAL) system $^{7}$ addresses the integration of digital libraries and archival technologies in support of stakeholders interested in studying important events. It focuses on events falling into two broad categories: (1) related to crises or tragedies as well as recovery and (2) government/community-related events (e.g., elections, demonstrations). The system monitors web-based (news, government, and other websites) and social media activity (tweets) to automatically detect interesting events and respond to general and specific requests archiving requests as well. Once events are identified, intelligent focused crawling and filtering approaches are used to ingest content and generate collections with high precision, recall, and low bias as is needed for scholarly study by social scientists. The system also incorporates a wide range of integrated services such as

\footnotetext{
6 http://learnweb.13s.uni-hannover.de

7 http://www.nsf.gov/awardsearch/showAward?AWD_ID= 1319578
}

browsing, searching, recommendation, clustering, sentiment analysis, summarization and visualization of data, information, or context.

The Big UK Domain Data for the Arts and Humanities (BUDDAH) project $^{8}$ aimed to develop a theoretical and methodological framework within which to study the archived UK web and web archives in general [19]. To demonstrate the value of web archives to humanities researchers and to build a community, a wide range of research projects was undertaken from analyzing disability action groups online to studying the Ministry of Defense's recruitment strategy. The project also worked on developing a suite of tools to support the analysis of the UK web archive and introduced an exploratory search interface SHINE $^{9}$ through which humanities scholars and social scientists accessed web archives [8].

The RESAW network, ${ }^{10}$ established in 2012, aims to establish and operate a collaborative world-class transnational European research infrastructure to enable cross-border studies of the archived web by integrating and opening up existing national web archives. It will facilitate easy access to large amounts of cultural heritage big data, as well as provide searching, selecting, and analysis of the material itself, thus making the research process more efficient and enhancing the European research area. At the moment, RESAW is a network of scholars and libraries interested in Web Archives and a research proposal to which we are contributing. We intend to make ArchiveWeb available to the participating libraries, both to get additional requirements from our partners as well as to support the collection building process for various national web archives.

\section{The Web Archiving Life Cycle Model}

Despite the increasing number of web archiving programs, best practices and a common methodology for web archiving have not been established yet [2]. The Web Archiving Life Cycle Model (WALCM), developed by experts at the Internet Archive, suggests a common framework for organizations seeking to archive the web. The model, reproduced in Fig. 1, includes several phases representing the various steps of a common workflow which organizations can refer to in order to create or improve their web archiving programs.

The original model is focused on the institutional policy collection; target users are archiving institutions

\footnotetext{
8 http://buddah.projects.history.ac.uk/

9 https://www.webarchive.org.uk/shine

10 http://resaw.eu/about/
} 
and curators of such collections. Each phase of this process is supported by the leading web archiving service Archive-It (mentioned above).

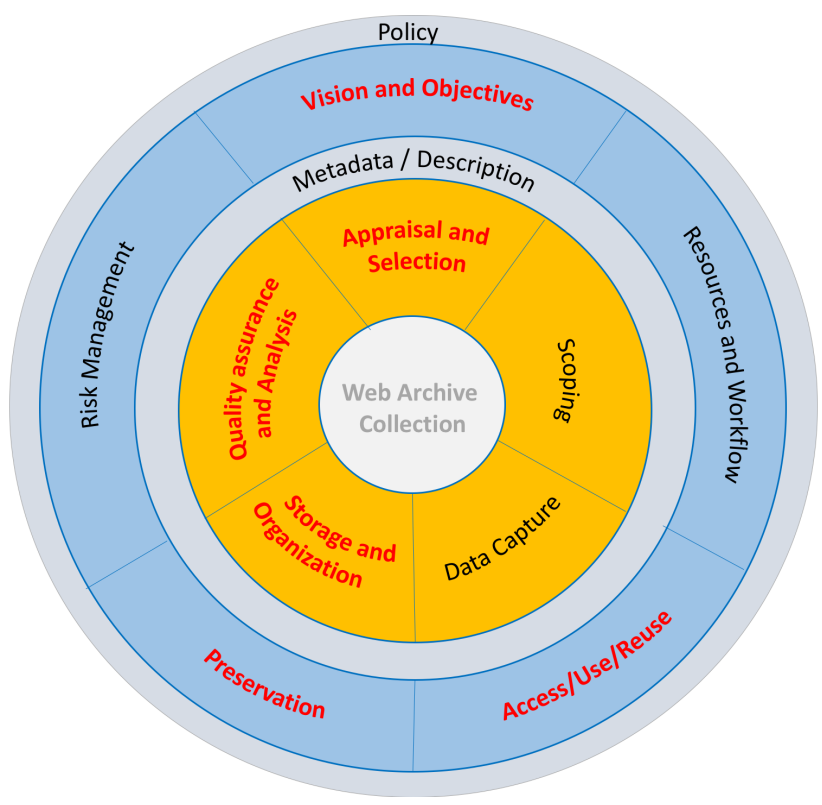

Fig. 1: Web Archiving Life Cycle Model

In the first phase of our work, we referred to the original WALCM model to prepare the investigation of current practices and of user requirements (Sect. 4). Figure 1 shows the steps of the WALCM model highlighted in red which we used as a reference to guide the semistructured interviews and to analyze the results. We focused on three steps related to high-level policy decisions (i.e., Vision and Objectives, Access/Use/Reuse, Preservation), and on three day-to-day tasks of the web archiving process (i.e., Appraisal and Selection, Storage and Organization, Quality Assurance and Analysis).

As regards the curatorial activities of storage and organization, and quality assurance and analysis, our interpretation is slightly different from the original one, though. With storage and organization we do not refer to the temporary or long-term storage plan of the organization, but rather we consider the possibility for ArchiveWeb users to organize resources in subcollections. Quality assurance and analysis is based on the possibility for ArchiveWeb users to collaboratively annotate and comment on resources in order to state or to discuss their relevance.

\section{User Requirements Analysis 2015}

\subsection{Motivation and Setup}

The motivation of our study was to provide a userfriendly interface to allow various kinds of users to access archived web collections and use the resources in various scenarios, e.g., for research or for learning.

In order to do so, in Summer 2015 we carried out a preliminary analysis of user requirements [6]. Participants were colleagues from the Internet Archive, as well as from libraries and similar institutions who are creating/curating Archive-It collections, e.g., Stanford University Libraries, Cornell University, University of Toronto, Columbia University, the National Museum of Women in the Arts, and the New York Arts Resources Consortium. The purpose of the study was to collect and analyze requirements for building and using web archive collections to be used as important input for new algorithms and tools to improve web archives. The ultimate goals of our work are to

- understand what kinds of web archive collections are being curated by different kinds of organizations

- contribute further improvements for working with web archive collections, especially regarding interface and search functionalities

With reference to the web archiving life cycle (Fig. 1 ), our interest was focused on specific steps related to policy decisions (e.g., Vision and Objectives, Access/Use/Reuse, Preservation), and data management (e.g., Appraisal and Selection, Storage and Organization, Quality Assurance and Analysis). Based on these six main categories, we used five leading questions to guide the semi-structured interviews:

1. What collections are built and what is the motivation for building these collections?

2. Who decides which collection is built and who is responsible for selecting which resources go into a specific collection?

3. Who are/could be potential users of a collection? Do you track the use of your collection?

4. Which functionalities are important for users who work with these collections when accessing Web Archive collections?

5. Are there any user interface improvements which you and your users already have on their wish list?

Respondents were free to add information and details related to their specific role/experience in the archiving process.

Interviews were conducted between August 3 and 6, 2015 , during a research visit of the investigators in San 
Francisco. We interviewed eight experts playing different roles and taking care of various activities in the archiving process, including the director of the Web archiving program at the Internet Archive, three Web archiving coordinators of University libraries, two Web archiving coordinators in national museums, one digital archivist, and one head of metadata services. The experts were selected based on suggestions provided by the Internet Archive. Three interviews were conducted in person at the Internet Archive premises; the remaining five were scheduled online according to the availability of the experts. Interviews took on average half an hour each, and the discussions were recorded for data analysis purposes. We used a consent form to inform the participants on the procedures and protection precautions and to collect their signatures for agreement.

Participation in the project was voluntary, and anybody was free to withdraw from the project at any point. Personal information is treated confidentially: Name and affiliations are anonymized in any resulting publications, unless participants give us explicit consent to identify them as a subject.

All recordings have been transcribed by one of the investigators, and qualitative content analysis of the transcripts has been carried out manually by two coders. Answers have been summarized referring to the categories in the original WALCM model (Fig. 1). In Table 1, we report a summary of the answers referring to the categories in the original WALCM model.

On the basis of this preliminary study, and building on previous work we did for collaborative learning environments [13], we designed and built the ArchiveWeb system to support collaborative creation and enrichment of web archive collections, with a focus on user interface and searching/sharing functionalities. We ingested 200 web archive collections from Archive-It, and asked our archiving partners for their input. In November 2015, we prepared a Memorandum of Understanding $(\mathrm{MoU})$ to describe how we integrate/use collections in ArchiveWeb, and we shared it with all participating institutions to collect their official agreement and signatures.

In the following, we briefly talk about some example collections, and in the next two sections, we describe the main ArchiveWeb functionalities and the evaluation provided by the experts who participated in the user survey.

\section{Web Archive Collections}

Archive-It is a subscription web archiving service from the Internet Archive that helps organizations to har- vest, build, and preserve collections of digital content. ${ }^{11}$ It was first deployed in 2006 and is widely used as a service to collect, catalog, and manage collections of archived web content. Full-text search is also available, even though effective ranking is still an open issue. All content is hosted and stored at the Internet Archive data centers.

Currently, the Archive-It system is mainly used by librarians and curators in order to build their collections. Less support is given to users and domain experts who actually want to work with the collections, or to the general public. ArchiveWeb supports searching, collecting, exploring, and discussing web archive collections such as the ones provided through the web archiving service of the Internet Archive.

Currently, about 200 collections from Archive-It have been integrated into the ArchiveWeb system with full metadata indexing, and can be explored through a visual interface. The Archive-It collections that were selected consist of those curated by organizations of the experts we interviewed as part of our user requirements analysis and those that cover a diverse range of topics such as Humanities, Arts, Society, Culture and Global Events.

In order to give a representative example of the diversity of the archived materials, we describe two specific collections.

\subsection{Human Rights Web Archive}

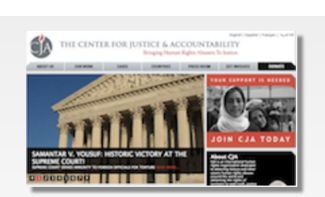

The collection Human Rights Web Archive ${ }^{12}$ (HRWA) by Columbia University Libraries is made up of searchable archived copies of websites Columbia University Human related to human rights created Rights Web Archive By Columbia University Libraries This collection is dedicated to preserving web- organizations, national human

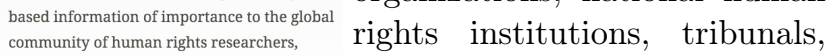
students, policymakers and advocates. and individuals. The collection was started in 2008 and is still

being continued, adding new websites on a regular basis.

Identification of websites for archiving is done by subject specialists with expertise in human rights and different regions of the world at Columbia University Libraries. Public nominations are provided by human rights researchers, advocates, organizations, and individuals who are involved in the creation of human rights-related websites. Priority is given to websites

\footnotetext{
11 https://archive-it.org/learn-more/

12 https://hrwa.cul.columbia.edu/about
} 


\begin{tabular}{|c|c|}
\hline Theme & Answers \\
\hline $\begin{array}{l}\text { Vision and Objectives } \\
\text { What collections are built and what } \\
\text { is the motivation for building these } \\
\text { collections? }\end{array}$ & $\begin{array}{l}\text { In most cases the motivation for starting the archiving of digital collections has been } \\
\text { to preserve: } \\
\text { - institutional or government websites } \\
\text { - special collections received as donations of materials from individuals or orga- } \\
\text { nizations } \\
\text { - event-based collections (e.g., Olympic games or election campaigns) } \\
\text { - research outputs (e.g., faculty members hosting their funded projects including } \\
\text { related assets such as documents and research datasets) } \\
\text { Larger institutions have a more structured approach including, in some cases, fo- } \\
\text { cused groups with their staff and researchers who determine which areas are in focus } \\
\text { with respect to priorities. For smaller institutions starting to archive online content } \\
\text { was also seen as a challenge }\end{array}$ \\
\hline $\begin{array}{l}\text { Access/Use/Reuse } \\
\text { Who are/could be potential users of } \\
\text { a collection? Do you track the use } \\
\text { of your collection? }\end{array}$ & $\begin{array}{l}\text { In most cases users are members of the library or of the university (e.g., professors } \\
\text { or researchers). } \\
\text { For specific collections, most of the requests to access archived web collections come } \\
\text { from people who are looking for documents that have been removed from a govern- } \\
\text { ment website. } \\
\text { Very few institutions and libraries track what users do using basic metrics (e.g., } \\
\text { number of hits on a collection level). }\end{array}$ \\
\hline $\begin{array}{l}\text { Preservation } \\
\text { What kind of websites should be } \\
\text { preserved? }\end{array}$ & $\begin{array}{l}\text { As regards to preservation, the priorities are to: } \\
\text { - collect non-governmental organizations websites as opposed to the government } \\
\text { websites } \\
\text { - preserve resources which are in danger of disappearing from the web }\end{array}$ \\
\hline $\begin{array}{l}\text { Appraisal and Selection } \\
\text { Who decides which collection is } \\
\text { built and who is responsible for } \\
\text { selecting which resources go into a } \\
\text { specific collection? }\end{array}$ & $\begin{array}{l}\text { All respondents confirmed that the current efforts at their institutions are mainly in- } \\
\text { dividual curators or subject specialists who are responsible for individual collections } \\
\text { or individual archiving requests } \\
\text { - in some cases a collection development executive group is appointed } \\
\text { - few institutions are collecting nominations from experts, researchers, library and } \\
\text { curatorial staff } \\
\text { Digital collections at larger institutions are growing incrementally, starting for ex- } \\
\text { ample from collecting the institutional memory, and including websites of single } \\
\text { departments and faculties, student organization websites, or special collections on } \\
\text { specific areas of interest. Institutions dealing with art (e.g., museums and galleries) } \\
\text { are particularly concerned with preserving resources which are in danger of disap- } \\
\text { pearing from the web. }\end{array}$ \\
\hline $\begin{array}{l}\text { Storage and Organization } \\
\text { Which functionalities are important } \\
\text { for users who work with these } \\
\text { collections when accessing Web } \\
\text { Archive collections? }\end{array}$ & $\begin{array}{l}\text { For curators } \\
\text { Collaboration is an important aspect in order to avoid duplicating resources and } \\
\text { efforts. Some curators are trying to find ways of collaborating in terms of } \\
\text { - collecting and displaying information } \\
\text { - creating and sharing metadata schema } \\
\text { - sharing folksonomies and tagging } \\
\text { Other useful functionalities would be: } \\
\text { - limiting the content to a certain number of seeds } \\
\text { - downloading metadata } \\
\text { - providing solutions to archive dynamic web pages } \\
\text { For final users } \\
\text { - Fulltext search } \\
\text { - Annotation } \\
\text { - Presentational functionalities (e.g., topic modeling, thumbnails of the major } \\
\quad \text { versions of the homepage per seed, link labels analysis }\end{array}$ \\
\hline $\begin{array}{l}\text { Quality Assurances and Analysis } \\
\text { Are there any user interface } \\
\text { improvements which you and your } \\
\text { users already have on their wish } \\
\text { list? }\end{array}$ & $\begin{array}{l}\text { Improvement of the interface } \\
\text { - Longitudinal data exploration } \\
\text { - query of subcollections } \\
\text { - integration of web archive resources into the general digital environment }\end{array}$ \\
\hline
\end{tabular}

Table 1: User Requirements Analysis - Summary of responses 
hosted in countries that do not have any systematic web archiving initiatives in place. Websites of intergovernmental organizations such as the United Nations are not included in the collection.

Archive-It services are used to maintain the collection, and the Internet Archive and Columbia University Libraries store copies of the resulting data. The collection includes over 711 websites with more than 50 million searchable documents and over 115 million archived documents with an archived data size of more than $5 T B$.

The HRWA collection provides a good balance between websites of large and well-known human rights organizations based in North America and Europe and websites of smaller organizations from other regions. One example of a website that no longer has a live version but can still be accessed by researchers via the Web archive collection is TibetInfoNet, which monitored the situation in Tibet http://www.tibetinfonet.net/. This page has been captured between May 2008 and July 2015.

The ArchiveWeb screenshot shows the human rights group restricted to "Tibet" -related resources, with the TibetInfoNet resource on the right side (Fig. 2). The URL provided in ArchiveWeb below the screenshot on the right side points to the original website, which is no longer available on the live web, but can be retrieved in its previous versions through the Wayback Machine (as described in Sect. 6.4).

\subsection{Contemporary Women Artists on the Web}

The collection Contemporary Women Artists on the $W_{e} b^{13}$ has been collected by the National Museum of Women in the Arts (NMWA) since December 2011. The NMWA is a gender-specific museum, located in Washington, and is the only museum solely dedicated to celebrating women's achievements in the visual, performing, and literary arts. Since opening its doors in 1987, the museum has acquired a collection of more than 4,500 paintings, sculptures, and works on paper and decorative art.

The collection Contemporary Women Artists on the $W e b$ includes three components: (1) Individual websites created by artists working in conceptual or new media art, (2) Artist profiles of women artists represented by contemporary art galleries, and (3) Women artist organizations.

One representative example of a resource in this collection is http://jodi.org, see Fig. 3, which has been

\footnotetext{
13 https://archive-it.org/collections/2973
}

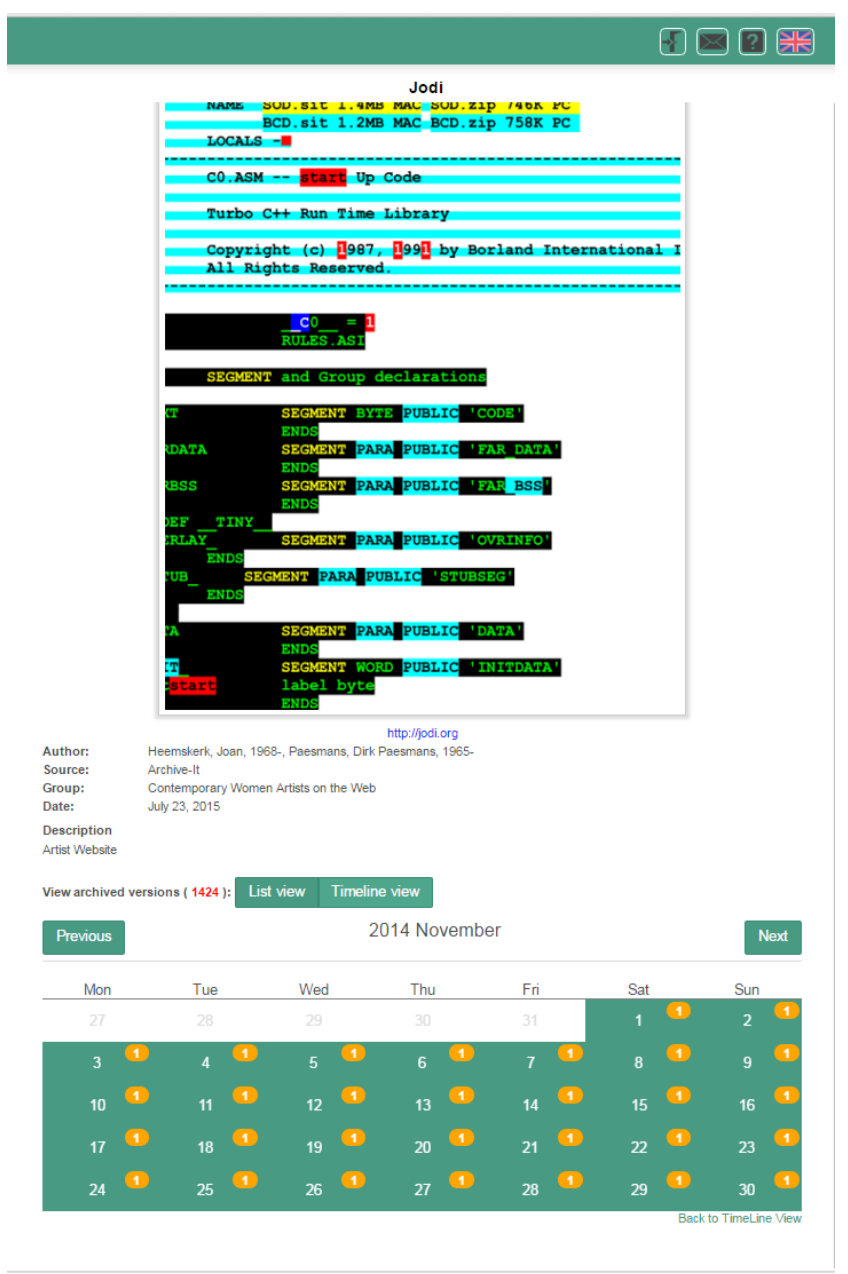

Fig. 3: Jodi Art Collective

captured 1418 times between April 16, 2009, and March 31, 2016, and which includes 726 videos.

As described in Wikipedia, ${ }^{14}$ it is the result of the initiative of two authors who work together toward a shared aim: Joan Heemskerk (born in 1968 in Kaatsheuvel, the Netherlands) and Dirk Paesmans (born in 1965 in Brussels, Belgium). Their background is in photography and video art. Since the mid-1990s, they started to create original artworks for the World Wide Web, and a few years later, they turned to software art and began the practice of modifying old video games to create a new set of art games [17].

Opening windows cascade across the screen, error messages squawk, and files replicate themselves endlessly. As graphics explode across the screen, the viewer gradually realizes that what had initially appeared to be a computer glitch is in reality the work of an irrational, playful, or crazed human [9]. Webpages are

\footnotetext{
14 https://en.wikipedia.org/wiki/Jodi_(art_ collective)
} 


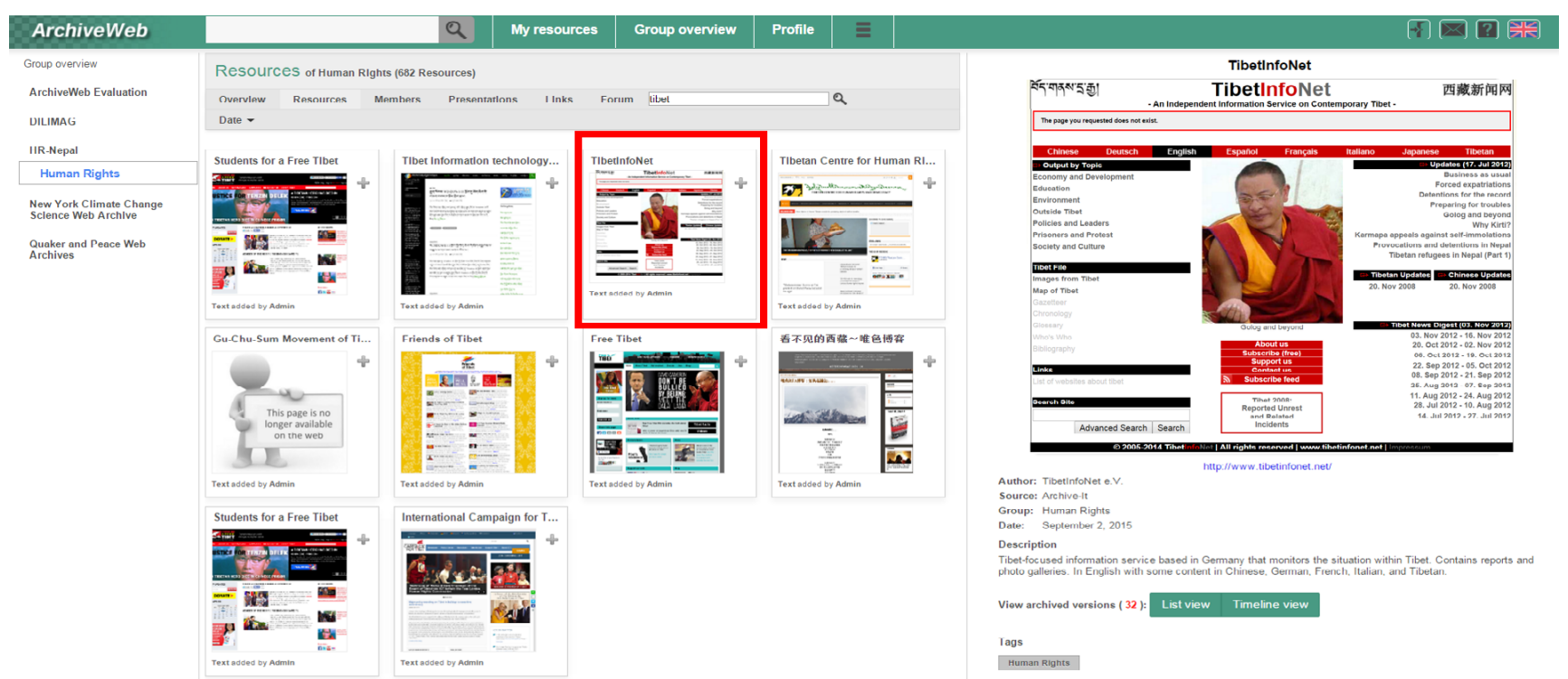

Fig. 2: Human Rights Example Resource

interactive, and a new perspective is displayed as the user clicks on various elements on the page.

Each day the webpage http://jodi.org changes, and a new piece of art is displayed. If the resource was not archived, all work would be lost. For this reason, it is important to archive at least a capture once a day in order to document the work of these artists over time.

\section{ArchiveWeb System: Main Functionalities}

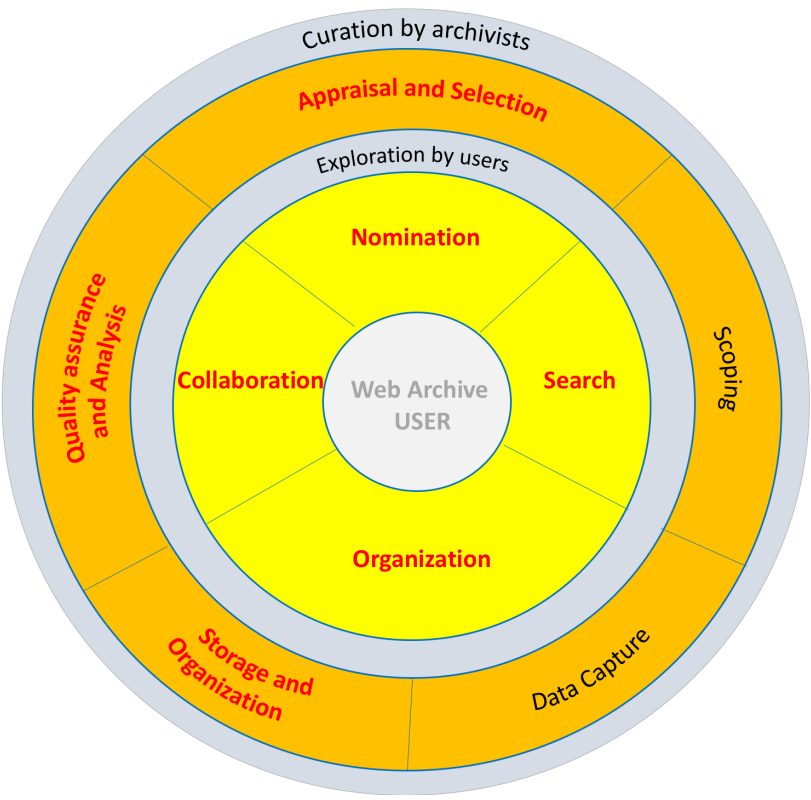

Fig. 4: The ArchiveWeb User Model
With the requirements described in Sect. 4 and the collections sketched in Sect. 5 in mind, we worked on providing the ArchiveWeb system to web archivists and libraries to facilitate collaborative exploration of multiple focused web archive collections.

At the design level, in ArchiveWeb we did not want to replicate services offered by the Archive-It service such as scoping, data capture, or risk management. Rather than focusing on the institution and collection level, we focus more on the final user who wants to explore and work with web archive collections. Thus, Fig. 4 highlights a more detailed inner circle with focus on the Web Archive User (instead of on the collections). Besides the curatorial tasks already present in the original WALCM model (i.e., Appraisal and Selection, Storage and Organization, Quality Assurance and Analysis) we detail four activities related to collaborative search and exploration of resources, as well as organizational functionalities (i.e., Nomination, Search, Organization, and Collaboration).

We used these categories, highlighted in red in the model, to guide the design of the system functionalities, as well as the evaluation of the system in 2016 (Sect. 7).

The main features provided by ArchiveWeb to support this exploration are: search - searching across multiple collections in conjunction with the live web, organization - grouping of resources for creating, merging or expanding collections from existing ones and collaboration - enrichment of resources in existing collections using comments and tags for the purpose of discussion. The following sections give a detailed description of these main system functionalities. In Sect. 6.1 we 
describe the technical workflow of the system, first describing how the data were collected and then how the system was implemented.

\subsection{Technical Workflow}

Data Collection Process. The Archive-It collection data was obtained by scraping the Archive-It website ${ }^{15}$. We selected a subset of collections to be crawled based on the subject metadata field that included the topics: Society \& Culture, Arts \& Humanities, Universities \& Libraries. Also collections specifically curated by the evaluators institutions were included in the crawl.

For each Archive-It collection, we collect and store the following metadata: collection title, collecting institution, description, subject, and collectors. All results/seed URLs displayed on each Archive-It collection page (e.g., Human Rights collection ${ }^{16}$ ) are also crawled. For each result, we store the following metadata: URL, title, description, subject, collector, creator, publisher, language, format, and type. If a result does not have metadata for title, subject, or language in Archive-It, we fetch the title, language, and subject from the metakeywords if available from the HTML page of the result. While crawling each result of a collection, we store the individual capture information fetched using the TimeMap component of the Memento framework. A TimeMap is a list of URIs of captures of the original result/URL (e.g., 4genderjustice.org ${ }^{17}$ ).

We update the Archive-It collections to keep ArchiveWeb content up to date with the information in the Archive-It portal by crawling and updating only those collections that have any result that has been captured/crawled recently (3 months before current time of crawl).

Implementation. The interface is implemented using the MVC framework JavaServer Faces (JSF) and PrimeFaces UI component library for JSF. Navigation across the ArchiveWeb system is facilitated through a grid layout in order to guide and keep the user in context and engaged at all times. For more details about the implementation and conceptual design of the initial platform LearnWeb on which the ArchiveWeb system has been built, we refer the reader to [13].

In the following, we discuss the extensions that we incorporated during the first year of the project in order to support the main features of ArchiveWeb. To provide

\footnotetext{
15 https://archive-it.org/explore/?show=Collections

16 https://archive-it.org/collections/1475

17 https://wayback.archive-it.org/1475/timemap/link/ http://4genderjustice.org/
}

access to the crawled Archive-It data from the abovementioned process, they have to be modeled as digital objects (resources, groups/collections) and stored in a relational database format, using the MariaDB repository. The data in the collections are made searchable through a metadata index, using Solr. Comments and tags added to resources by various collaborators while curating collections are also indexed in order to support collaborative search and exploration. Search filters based on the various metadata fields of the results were incorporated using the Solr faceted search feature to narrow down search results.

As each of the resources/URLs has a number of archived versions, the challenge was how to incorporate navigation through these versions in the interface. To support users to navigate through the different versions, a timeline view similar to the Wayback Machine interface was incorporated (see Fig. 8). A simple list view was also included to provide a one-step navigation for resources with very few archived versions. Finally, we also implemented a functionality for merging existing collections and creating new ones, so that users have the flexibility to merge multiple collections covering similar topics/subjects and build upon them.

\subsection{Search}

\begin{tabular}{|c|c|}
\hline \multicolumn{2}{|l|}{ Options } \\
\hline Add to: & $\begin{array}{l}\text { My resources } \\
\text { ArchiveWeb Evaluation } \\
\text { HR-Nepal }\end{array}$ \\
\hline Title: & Human rights - Wikipedia, the free encyclopedia \\
\hline Description: & $\begin{array}{l}\text { Human rights are moral principles or norms, that } \\
\text { describe certain standards of human behavior, and are } \\
\text { regularly protected as legal rights in municipal and ... }\end{array}$ \\
\hline $\begin{array}{l}\text { Url: } \\
\text { Type: } \\
\text { Source: } \\
\text { Captured between: }\end{array}$ & $\begin{array}{l}\text { https://en.wikipedia.org/wiki/Human_rights } \\
\text { text } \\
\text { Bing } \\
12 / 9 / 03 \text { and } 1 / 30 / 16\end{array}$ \\
\hline & Save \\
\hline
\end{tabular}

Fig. 5: Options Dialog

ArchiveWeb provides a keyword-based search system that returns results from ArchiveWeb collections as well as from the live web (Fig. 6) using the Bing Search API. ${ }^{18}$ ArchiveWeb collections includes ArchiveIt collections as well as user created collections stored in the system. If users search for a keyword (e.g., "human rights"), ArchiveWeb returns a list of results from these

18 http://datamarket.azure.com/dataset/bing/search 


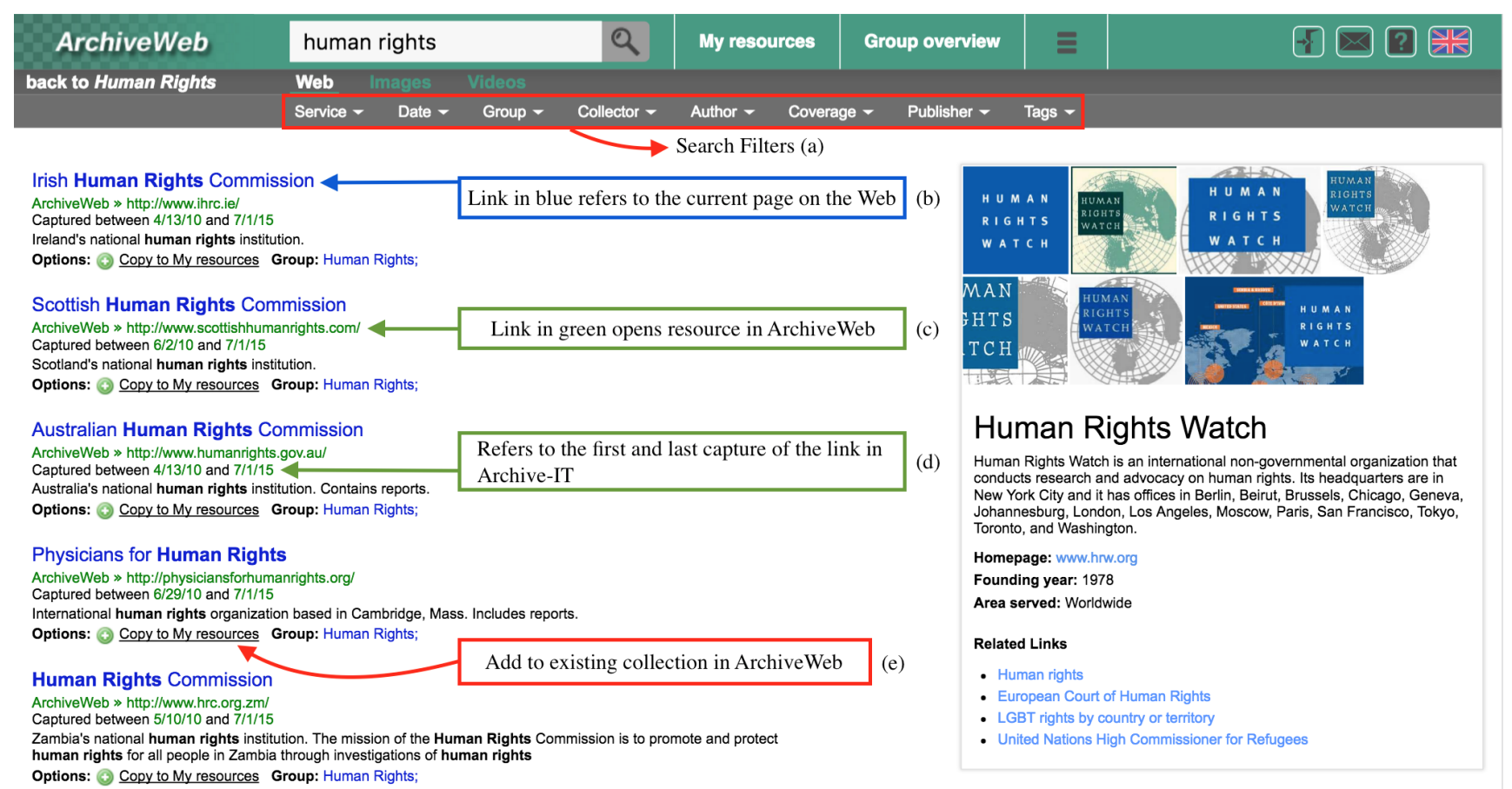

Fig. 6: Search Page

sources, indicating whether the resource comes from a specific Archive-It/ArchiveWeb collection or from the live web (Fig. 6b). The search results are presented in this manner, so that live web results which are not yet part of any collection could be added to existing ones. The results being returned from Archive-It collections are based on a full metadata index for these collections. A full-text index of all content is planned for the next version. Besides webpages, images (from Bing, Flickr, Ipernity) and videos (from YouTube, TED, Yovisto, Vimeo) can also be searched, but not yet from ArchiveIt collections. For Archive-It collections, each search result provides a pointer to the resource item saved in ArchiveWeb (Fig. 6c) and displays information about when it was captured in Archive-It (Fig. 6d). Live web results are not yet saved in ArchiveWeb but can be copied by the user into a group (Fig. 6e); in this case, the Options dialog box (Fig. 5) provides details about when/if this page has been indexed by the Internet Archive, retrieving the information from the Wayback CDX server API. ${ }^{19}$

Search results can be refined using faceted search filters visible below the search box (Fig. 7). For example, for our evaluation it was important to provide filters to show only the resources in a specific collection (Filter: Group) or that were archived by a specific institution (Filter: Collector).

\footnotetext{
19 https://github.com/internetarchive/wayback/tree/ master/wayback-cdx-server
}

\subsection{Organization}

ArchiveWeb provides the functionality to organize resources into collections (groups of resources) according to clearly defined and coherent themes/topics. This functionality allows working with existing groups, creating new collections/groups and sub-groups, adding new resources to groups, and moving resources between groups.

A group overview interface allows browsing through existing collections available within ArchiveWeb and the collections that a user has created or joined. Descriptions for every collection provide information about the topic/theme and what kind of resources it contains. For Archive-It collections, the description reports the details available on the public Archive-It interface along with a reference link to the specific collection in the Archive-It platform. It is possible to filter collections by searching through the title and description fields.

After joining specific groups/collections users have the opportunity to edit metadata of existing resources and to contribute new resources from other existing collections or from the live web. The ArchiveWeb collections, derived directly from their Archive-It counterparts, are "read only". Users can browse through the resources of each collection using the advanced visualization and exploration functionalities of ArchiveWeb, but they cannot change the original collection. They 


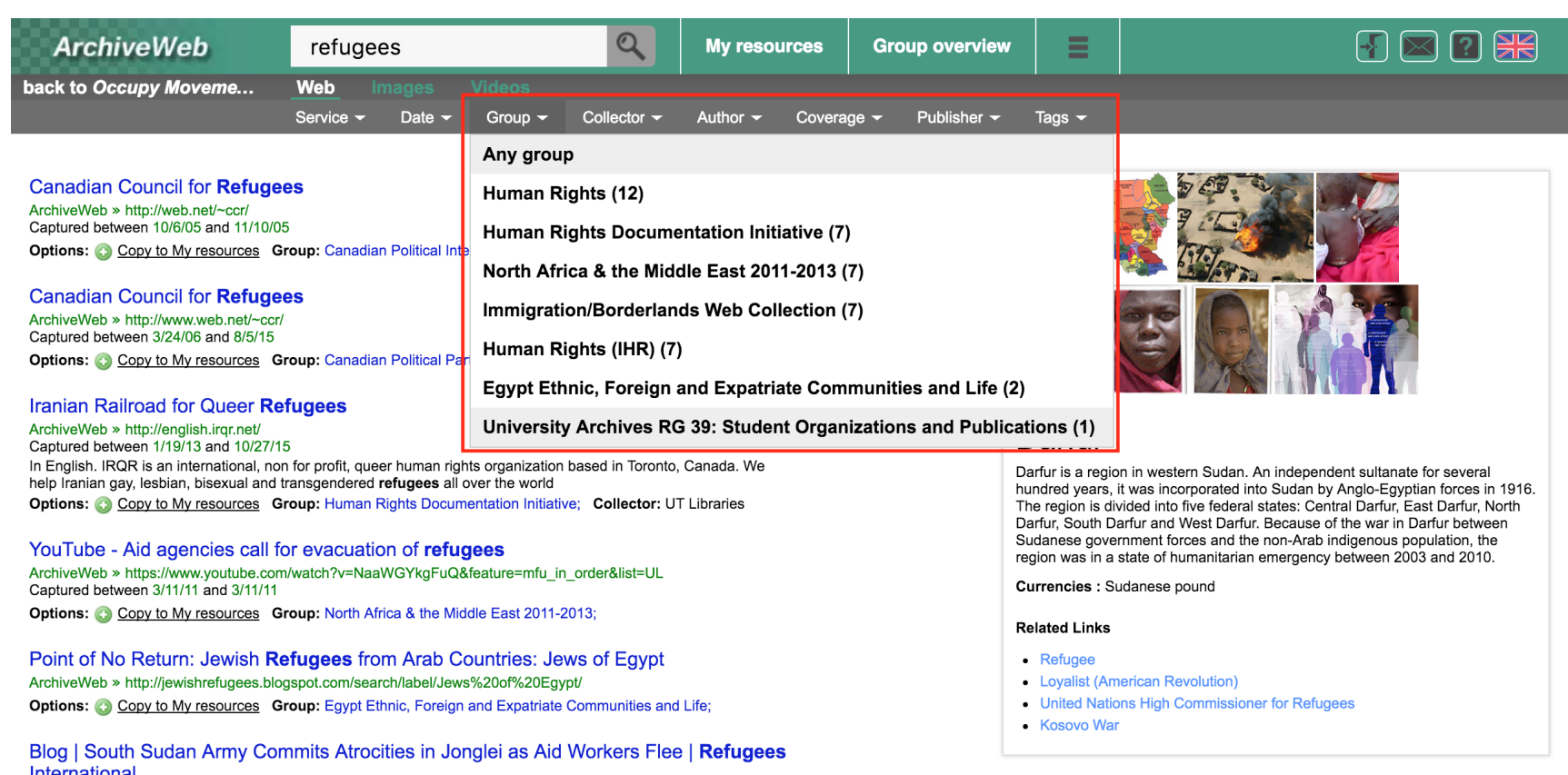

International

ArchiveWeb » http://refintl.org//blog/south-sudan-army-commits-atrocities-jonglei-aid-workers-flee/

Options: () Copy to My resources Group: North Africa \& the Middle East 2011-2013;

Fig. 7: Refinement through Faceted Search Filters

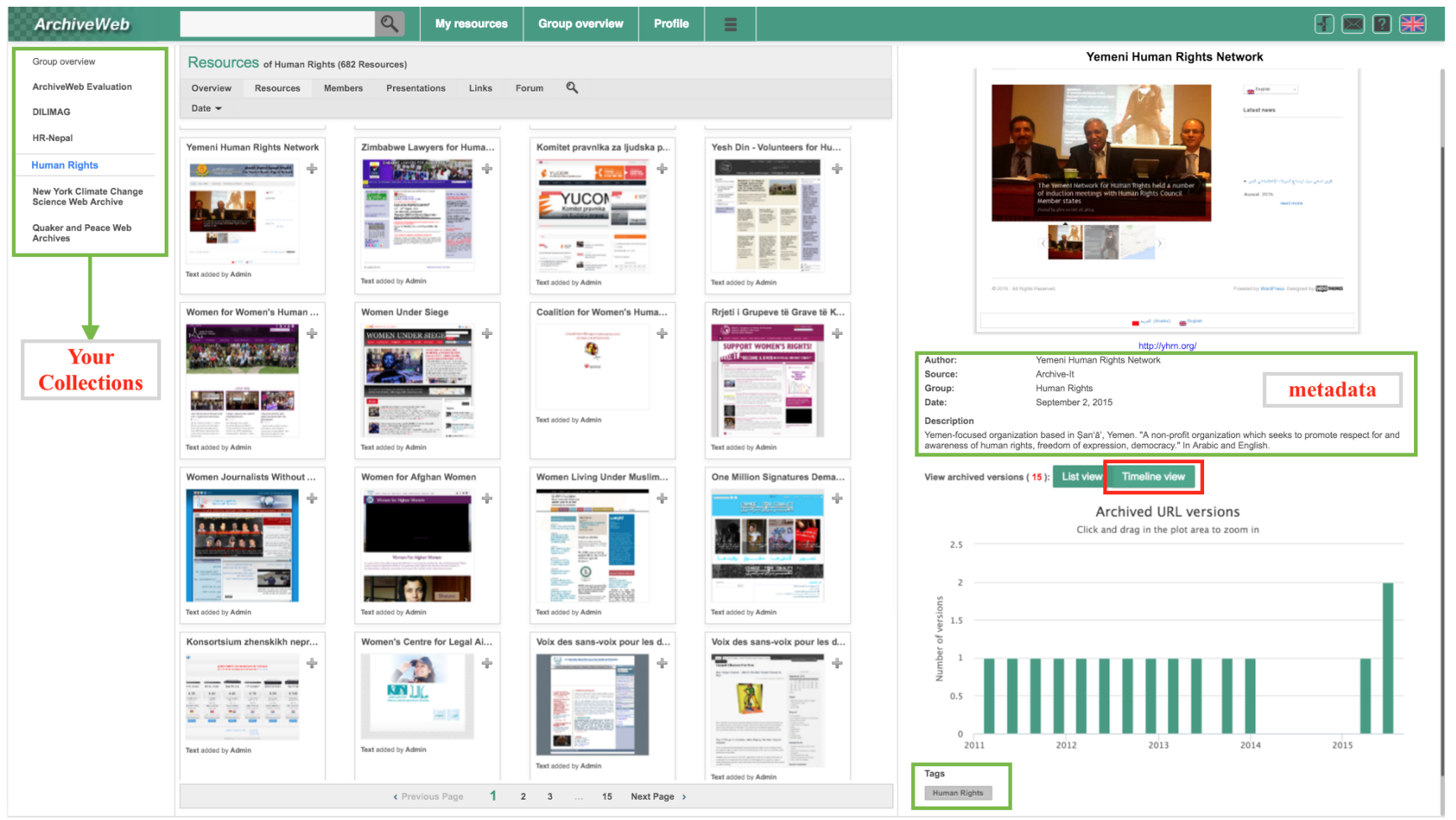

Fig. 8: Group Resources Interface

can create a copy of the entire "read-only" group or add individual resources to their own collections by se- lecting resources individually, as well as merge multiple existing collections into one new collection. 
Users can also organize their resources into subgroups within collections in order to group resources that are related to a similar subtopic. Resources can be uploaded to a collection either from the desktop or by suggesting a URL. Each group has a specific interface that visualizes all thumbnails of the most recent snapshot of each resource when it was added to the group (Fig. 8). Resources that no longer exist on the web, or do not have a redirect available, display a thumbnail with the message "The page is no longer available on the web" (we are currently implementing the functionality to upload a thumbnail of an earlier capture of the page taken from the Internet Archive Wayback Machine). The overview interface within a group provides a summary of the activities of various members of the group, including the actions of newly added resources, resources which were edited or deleted, and users who have joined/left the group.

\subsection{Collaboration}

After a resource is added to a ArchiveWeb collection, the resource can be enriched with additional comments and tags (in addition to the metadata already provided). The comments on a resource can be used to discuss why this resource has been chosen as a seed for a collection, to decide upon the crawl frequency and crawl depth, as well as any other issues and discussions which should be documented during the collection building process (Fig. 9). By exchanging comments, collaborators can also discuss and decide on the relevance of a suggested seed resource for a particular collection. ${ }^{20}$ The use of tags helps categorize or label a resource with subjects or topics covered by the resource, making it possible to browse collections by filtering based on certain tags. Users can also edit metadata such as title, description, and author fields.

During the collection building process, multiple collaborators can join a newly created ArchiveWeb collection and suggest seed URLs from the live web or from existing collections in ArchiveWeb. Collaborators can add comments and tags to these seed URLs as highlighted above to indicate the importance of this resource to the collection. Once the collaborators have finalized the seed URLs, they use the Archive-It system to curate this collection by specifying the seeds with corresponding metadata, crawl depth, and frequency. In the future, we will investigate a direct connection between ArchiveWeb and the Archive-It system, in order

20 ArchiveWeb is not (yet) directly coupled to the ArchiveIt administrative interface: curators have to switch to this interface to add suggested seed URLs to the corresponding Archive-It collection. to provide a seamless environment for working with and archiving Web collections.

The system allows users to archive a single page or a website (resource) by clicking on the "Archive Now" button which sends a request to the Wayback Machine to archive it. This functionality is similar to the "Save Page Now" feature of the Wayback machine, and it supports users to gather captures easily as they work within the system. All captures from Archive-It as well as the Wayback captures in ArchiveWeb added using the "Archive Now" functionality are visualized both as a list and in a timeline view to help users navigate through the different archived versions that are available. The timeline view visualizes a timeline displaying the number of archived versions aggregated by month for each resource. Figure 8 shows a timeline view on the bottom right.

\section{Evaluation and Feedback 2016}

At the end of 2015, the release of ArchiveWeb, which took into account the initial requirements collected in summer 2015, was ready for a first evaluation. In February 2016, we invited all experts, who provided the initial user requirements, to participate in a task-based evaluation of the system. All of them agreed to participate.

For the evaluation, we imported 200 publicly accessible web archive collections from Archive-It into ArchiveWeb in order to test the potential of the system to support collaborative work with such collections. The evaluation was done using a task-based evaluation design, analyzing both quantitative interaction data (usage logs) and qualitative feedback in the form of interviews. We asked the experts to carry out two sets of tasks: individual and collaborative. The individual tasks included creating a sub-collection from an existing collection of their institution, enriching an existing collection with additional relevant resources from the live web and annotating resources with information about why they should be included and how often they should be archived. The collaborative tasks involved selecting ten featured resources from their collections, including them into a joint group shared with all other evaluators, and discussing the reasons why such resources were included. The final task involved creating a new collection about a shared topic among the evaluators, searching for relevant seed URLs for this collection using ArchiveWeb, and agreeing with the rest of the evaluators about which seeds should be included, what should be the archiving frequency, and why.

The evaluation period spanned about three weeks, after which we invited the evaluators to give us feedback through a questionnaire and through follow-up in- 
terviews to fully understand their experience. In the following, we will summarize the feedback we received and point out specific interesting responses and suggestions, based on the questions we asked. The headings denote the questions being asked.

\subsection{Usage Log Analysis}

In the ArchiveWeb system, we log the various actions carried out by the user while working with the system. The types of actions logged include annotation (tags or comments added to resources), searching (queries issued, type of search such as image, video, webpages, search filters used), resource-level actions (additions/deletions/edits) and group-level actions (metadata edits/subfolders actions/search within a group/group merging).

After the evaluation, we analyzed the usage logs to understand how the evaluators worked with the ArchiveWeb system and how the various tasks were completed. For the initial collaborative task of selecting featured resources from their collections to be added to a shared group with the evaluators, 6 of the 8 evaluators participated in this task. Most of the evaluators added few featured resources from their own collections to the shared group, but one of the evaluators added resources directly from Bing by searching for known URLs, and one evaluator added resources that were previously uploaded to a newly created collection. All of the evaluators tagged their resources, and one evaluator added comments to discuss why a resource was included. None of the evaluators added comments on resources added by others; thus there was no discussion or collaboration between the evaluators. This could be due to different interests in topics among the evaluators. Evidence from the usage logs shows that the evaluators could not carry out the final collaborative task of creating a new shared collection based on a shared topic and collecting seed URLs due to the short time period of the evaluation and limited communication between the evaluators.

As specific examples, let us here summarize the individual tasks carried out by two evaluators as observed from the logs. A Web archiving coordinator of a university library initially joined his institution's various collections covering topics such as Human Rights, Arts \& Humanities, Society \& Culture. He then created a new collection by merging resources from two existing collections. Later, he carried out 20 searches related to climate justice or urban planning with the use of filters such as "author", "tags", "groups". He added tags and comments related to crawling strategies of resources such as "one-time capture". Finally, he worked for 50 min on the initial collaborative task and added 10 featured resources to the joint group with comments discussing their importance.

A Web archiving coordinator of a national museum created a new collection and added about 10 resources from an existing collection of her institution. Then she carried out 5 searches related to photography/ photography galleries using filters such as "service" or "groups" and added 5 resources from Bing to a sub-folder created within the new collection. Later, she added tags and comments such as "photo gallery" and "archive monthly". Finally, she searched for 5 known resources from Bing and added them along with 3 other resources from an existing collection to the joint group with tags, in order to get a feel of how close the workflow of ArchiveWeb was to the existing one. This final task was completed in $20 \mathrm{~min}$.

\subsection{Evaluation: Questions and Answers}

As we mentioned in Sect. 4, one of the goals of our work is to contribute further improvements for working with web archive collections, especially regarding collaborative search and exploration of resources, as well as organizational functionalities. For this reason, we asked the evaluators to give us feedback about the current functionalities, as well as suggestions for further improvement. In general, all evaluators appreciated the curatorial functionalities provided by the system. The answers were analyzed manually and are categorized according to the four main steps reported in the ArchiveWeb User Model in Fig. 4.

\subsubsection{Nomination}

Seed URL Discovery. The integration of searching across web archive collections as well as the live web, providing the ability to suggest additional material that could be archived, is a new feature which is not present in existing systems used for curation/creation of web archive collections, and it was much appreciated by the evaluators: I really liked the feature of searching the web in conjunction with the web archive collections available in the system, an entirely new feature which was seamless and straightforward.

Others positively stressed the possibility to archive web pages directly from the interface: I also like that the "Archive Now" feature is so well embedded in the platform - it will make it very easy for people to gather new captures as they work, without having to leave the interface. 
Nomination tool. Nomination for collaborative collection building was mentioned as well: A nomination function would be nice - like URL suggestion instead of having to upload all resources. ... The inclusion of screenshots and ability to pull in resources from live web could make this tool a better option than the UNT nomination tool ${ }^{21}$ for collaborative collection building. ... Ability for non-members to submit tags for content or suggest content for future archiving could be submitted for approval first.

\subsubsection{Search}

A few remarks concerned our ranking of results and included suggestions for (i) providing a transparent explanation of how the relevance ranking is determined, (ii) providing the total number of captures of a page along with the capturing period in order to determine the popularity of an archived result, and (iii) cues as to how many results were returned. Users in general liked the information provided on the results page: Yes, I think the highlighted search term and snippet are sufficient for each item in search results. I like that the web archive hits are placed before the live web hits, but others also mentioned: The results would provide enough information if the archived resources had rich enough metadata. It would make way more sense if there were more tags.

A related suggestion concerned results from different sources. Users liked the possibility to retrieve results from several sources: The search results seem to offer enough information. I appreciate that the user can filter by the source of the content (e.g., Archive-It, ArchiveWeb, Bing), but others asked for more clearly separating results from different sources or defaulting to particular sources: I do wonder about the utility of having the Bing results mixed with the Archive-It results unless the idea is that the user will use these results to add resources to collection ... I would think that the default search results would just be Archive-It collections then with the opportunity to expand out next.

Other remarks asked for advanced search functionalities such as limit to collection, limit to domain/path, search within title only. The experts also requested fulltext search of Archive-It records (WARCs) instead of just metadata search.

\subsubsection{Organization}

Efficiency through the interface. From conversations with Archive-It partners and the NDSA Web Archiving Survey, it became clear that web archiving on the whole

\footnotetext{
21 http://digital2.library.unt.edu/nomination/
}

takes a lot of time to do well. Therefore, for curatorial needs, efficiency is a critical issue. This asks for making it as quick and intuitive as possible to move from one task to another and making tasks as efficient as possible (providing bulk operations, summary views, etc.)

One of the evaluators stated that: ArchiveWeb increases curatorial functionality over that of Archive-It, e.g., on-the-fly creation of groups, moving resources between groups, and easily annotating resources.

Collection management. Several of the new functionalities of ArchiveWeb were positively highlighted by the evaluators including (i) the ability to curate new arbitrary collections of seed records from across multiple web archive collections, (ii) the possibility of having resources exist at multiple levels (e.g., personal, group, sub-folder), and (iii) the ability to create collaborative collections with colleagues from various institutions.

Improving the user interface. A few remarks were specifically referring to curatorial functionalities, such as bulk operations: selection of multiple resources either by clicking, searching, or filtering and then adding/moving to a collection; collective tagging of multiple seed records; and initiating archiving for multiple seed records. One of the evaluators mentioned: $I t$ ' $d$ be great to tag similar collections and allow them to be grouped together without having to create a new collection.

An undo feature would also be useful for ArchiveWeb: I would like to see some sort of "undo" feature for mistakes that are made in the system (for example, if I deleted something from a group and immediately felt it was in error, I could undo that action).

Exporting resources and metadata. Several experts missed a way for exporting resources and metadata for research / usage outside of ArchiveWeb, and means to request derivative data from Archive Research Services such as WATs ${ }^{22}$, WANEs ${ }^{23}$, LGAs ${ }^{24}$, etc.: I would appreciate means of interacting with content instead of just searching for web pages, e.g., ngrams, word frequency like UK Web Archives SHINE ${ }^{25}$.

22 Web Archive Transformation (WAT) files contain key metadata such as capture information, essential text and link data, and other information extracted from (W)ARC files.

23 Web Archive Named Entities (WANE) files contain a list of people, places and organizations mentioned in each valid archived record, extracted using Stanford Named Entity Recognizer.

24 Longitudinal Graph Analysis (LGA) files feature a complete list of what URLs link to what URLs, along with a timestamp, within an entire web archive collection.

25 https://www. webarchive.org.uk/shine 
Experts liked the ability of our interface to provide an easy overview over resources and collections using screenshots, but also suggested to provide screenshots of different captures and select a specific capture as default screenshot (the default we implemented for the evaluation was the screenshot of the last available page).

\subsubsection{Collaboration}

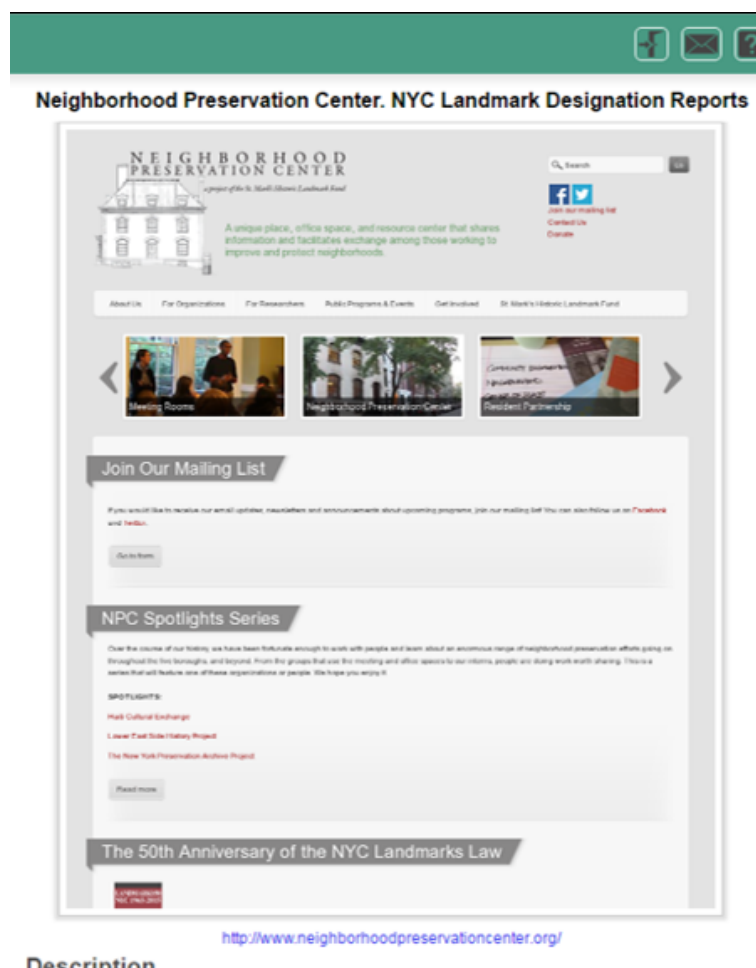

This database will soon house printable PDF files of the entire collection of NYC Landmark Designation Reports. Reports are entered based on the year a property was designated as a Landmark and the earliest reports are now available. NOTE: Archived reports accessible only through "view all" link.

\section{Current version: Archive now}

\section{View archived versions ( 15 ): List view $\quad$ Timeline view}

\section{Tags}

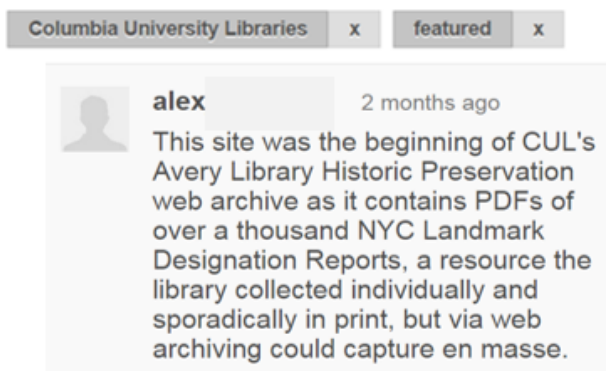

Fig. 9: Resource annotation
The evaluators positively highlighted the usefulness of ArchiveWeb to facilitate collaborative collection building and to make web archives more useful to researchers: (i) It is a really fun tool to use! ... I enjoyed using ArchiveWeb and think it has great potential in facilitating collaborative endeavors and potentially collection building, (ii) I think it's incredibly easy to use. Institutions, particularly those without a great deal of IT support, would really benefit from having an interface like this to help entice users to interact with web archive collections. One of the most exciting aspects of this project is the potential for users to help us build and enrich collections through suggesting resources and annotating what we've already archived. I'm excited to see how this project progresses.

Collection Enrichment. One of the new functionalities that was positively highlighted by the evaluators is the possibility to add schema-agnostic classification and other information using tags. One participant (Technical Manager) asked for more metadata fields for newly added resources besides title, author and description, to make it easier for curators from different institutions to collaboratively build collections: Tagging functionality is helpful, but not quite same as being able to define a custom field name and supply a value for that field.

Other appreciated functionalities were (i) the ability to use notes/comments in order to highlight the motivations for collecting (Fig. 9): I also have not previously been able to tag or annotate resources within collections (Archive-It does allow for adding notes in their new release, but these are only useful as an administrator of an account and not to the public/user), and (ii) the display of the capture frequency, highly useful when developing collaborative collections across institutions.

Regarding annotations, suggestions included autosuggestion of tags from within the same collection, optionally allowing tag suggestions from controlled vocabularies, and conducting more robust mining of the text of archived resources to suggest possible annotations, plus the possibility to include external contributors for tagging: It'd be great to turn on external tagging, or pick certain trusted external users to tag.

Finally, we asked for further suggestions on any topic important to the users.

Many evaluators found the system rather intuitive, but had to refer to the documentation about the functionalities that we had provided in order to feel comfortable using the system: (i) The PDF instructions helped since there are not a lot of "helpers" or "tool tips" in the interface itself. More help features baked into the interface would help as not all the features are well defined or obvious. (ii) Additional text could be useful to 
users - I would suggest hover text, where the user holds the mouse over something and a description would pop up, with an option to close it and not see it again, if desired. Also, having information about the model underlying the metadata (schema, ontology) could help understand how this metadata could be shared with other systems.

One of the experts suggested that for users who are unfamiliar with web archives, it would be good to provide some information highlighting the need and challenges of web archives: I think users unfamiliar with web archives might need some tools or modules or something to help better understand some of the challenges of web archives. Otherwise, they may just think of web archives as a "blackbox" and accept collections uncritically, unaware of why some things work and some things don't. That could just be something like a mouse over or an FAQ entry though.

Table 2 summarizes the results of the evaluation, with particular attention on the phases supported by the ArchiveWeb User Model.

In the next iteration of the evaluation, we will focus specifically on collaborative tasks, in order to clearly understand the collaborative potential of the system for Web archiving. The planned evaluation will span 12 months in order to give our partners more time to collaboratively discuss and build a collection together.

\section{Future Improvements in the Next Release}

Based on the suggestions provided by the evaluators, we plan to incorporate the following new functionalities in the next release, in addition to several smaller user interface improvements asked for during the evaluation:

Thumbnail Visualization. We are working on a specific visualization to display the thumbnails for different captures of an individual resource/seed, where the curator/user can choose how many different thumbnails he wants to view.

Search Result Aggregation. We are also working on a new search interface design that aggregates results from different sources, displaying some representative examples for each source, and in addition will allow users to select a default source for which we display the results in detail, i.e., using a list of results view similar to the current design.

Bulk Operations. We will also work on bulk operations which allow users to select multiple resources by clicking, searching, or filtering and then adding them to a collection. The system will also support bulk editing of resources within a collection such as collective tagging (e.g., to assign a tag to multiple seed records), as well as tagging similar resources across multiple collections and then allowing the option to group them together into a new collection.

Exporting Resources. We will also implement the ability to export resources and metadata from collections for research or usage outside of ArchiveWeb, for registered users who are allowed to use this export functionality (usage will have to be restricted because of potential copyright issues).

Advanced Search. We will extend our advanced search and faceted search options to limit search within certain collections, specific domains, or paths, and to search within title or description only.

Fulltext Search. We will also incorporate full-text search of Archive-It websites instead of just the metadata, even though not all evaluators even realized that this functionality was not yet provided, as rich metadata descriptions are available for many collections. Obviously, this fulltext search capability will have to go along with appropriate ranking optimizations in order to take into account the longitudinal character of Archive-It collections, which usually include many captures per resource which have to be considered.

Collaborative Aspects. We will allow external contributors to suggest tags, which can then be approved by the curators of the collection. The system will also support the possibility to invite collaborators to join collections from within ArchiveWeb.

\section{Conclusions}

In this paper, we discussed the ArchiveWeb system which supports collaborative exploration of web archive collections. We provided a description of the main features of the system such as (i) searching across multiple collections as well as the live web, (ii) grouping of resources for creating new collections or merging existing ones, as well as (iii) collaborative enrichment of resources using comments and tags.

The system has been developed based on an iterative evaluation-driven design-based research approach. Starting from a platform which already supported collaborative search and sharing of web resources, ArchiveWeb was designed to address web archive expert users' requirements (librarians and curators in archiving institutions), as described in Sect. 4. The resulting 


\begin{tabular}{|c|c|}
\hline Current Functionalities & Required Functionalities \\
\hline \multicolumn{2}{|l|}{ Nomination } \\
\hline $\begin{array}{l}\text { - Seed URL Discovery: ability to curate new arbitrary col- } \\
\text { lections of seed records from across multiple web archive } \\
\text { collections } \\
\text { - Possibility to archive web pages directly from the interface }\end{array}$ & $\begin{array}{l}\text { - A "nomination" function would be nice - like URL sug- } \\
\text { gestion - instead of having to upload all resources } \\
\text { - Display the capture frequency }\end{array}$ \\
\hline \multicolumn{2}{|l|}{ Search } \\
\hline $\begin{array}{l}\text { - Combined searching of seed metadata with search engine } \\
\text { of live web } \\
\text { - Dynamic effects of "List View" and "Timeline View" visu- } \\
\text { alizations for individual seed records }\end{array}$ & $\begin{array}{l}\text { - Full text search of WARC } \\
\text { - Google as well as Bing as web search options } \\
\text { - Advanced search options (e.g., limit to collection, limit to } \\
\text { domain/path, search within title only) } \\
\text { - Provide more details about the total number of captures } \\
\text { of a page along with the capturing period } \\
\text { - Means of interacting with content instead of just searching } \\
\text { for web pages (e.g., ngrams, word frequency) }\end{array}$ \\
\hline \multicolumn{2}{|l|}{ Organization } \\
\hline $\begin{array}{l}\text { - On-the-fly creation of groups (sub-collections) } \\
\text { - Possibility of having resources exist at multiple levels } \\
\text { (e.g., personal, group, subfolder) }\end{array}$ & $\begin{array}{l}\text { - Bulk operations: select multiple resources and work with } \\
\text { all at once (e.g., copy, move, edit, group, delete, annotate, } \\
\text { share) } \\
\text { - Exporting of resources and metadata for research/usage } \\
\text { outside of ArchiveWeb } \\
\text { - Provide information about the model underlying the } \\
\text { metadata }\end{array}$ \\
\hline \multicolumn{2}{|l|}{ Collaboration } \\
\hline $\begin{array}{l}\text { - Seed collection: ability to share seed URLs across collec- } \\
\text { tions with colleagues from various institutions } \\
\text { - Annotation: ability to use notes/comments in order to } \\
\text { highlight the motivations for collecting } \\
\text { - Forum for users to discuss collections }\end{array}$ & $\begin{array}{l}\text { - Collection Enrichment: possibility to add schema-agnostic } \\
\text { classification and other information using tags } \\
\text { - Request collaborators within the system } \\
\text { - Allow external contributors for tagging (could be submit- } \\
\text { ted for approval first) }\end{array}$ \\
\hline
\end{tabular}

Table 2: System Evaluation - Summary of results

ArchiveWeb system, fully functional and publicly available, ${ }^{26}$ has been evaluated through a task-based evaluation study carried out with the same experts who participated in the preliminary investigation. After the quantitative analysis of the logs and the qualitative feedback from the evaluation, we are now incorporating new features such as exporting resources, bulk editing operations, new visualizations, and advanced search, which will be available in the next release of the system.

Acknowledgements We especially thank Jefferson Bailey from the Internet Archive who provided us with the contacts to his colleagues at university libraries and archiving institutions, and for his helpful comments during the requirements and evaluation phase. We are also grateful to all experts, who participated with enthusiasm in our evaluation, providing valuable feedback and useful suggestions to improve the ArchiveWeb system. This work was partially funded by

26 http://archiveweb.13s.uni-hannover.de/aw/index. jsf the European Commission in the context of the Alexandria project (ERC advanced Grant No. 339233).

\section{References}

1. Alonso, O., Strötgen, J., Baeza-Yates, R., Gertz, M.: Temporal information retrieval: challenges and opportunities. In: Proceedings of the 1st international temporal web analytics workshop (TWAW 2011) associated to WWW'11, pp. 1-8 (2011)

2. Bragg, M., Hanna, K., Donovan, L., Hukill, G., Peterson, A.: The Web Archiving Life Cycle Model. White Paper (2013). Available at http://ait.blog.archive. org/files/2014/04/archiveit_life_cycle_model.pdf

3. Cutrell, E., Robbins, D., Dumais, S., Sarin, R.: Fast, Flexible Filtering with Phlat. In: Proceedings of the SIGCHI Conference on Human Factors in Computing Systems (CHI '06), pp. 261-270 (2006)

4. Dougherty, M., van den Heuvel, C.: Historical infrastructures for web archiving: Annotation of ephemeral collections for researchers and cultural heritage institutions (2009). Available at http://web.mit.edu/commforum/mit6/papers/Dougherty_Heuvel.pdf 
5. Dumais, S., Cutrell, E., Cadiz, J., Jancke, G., Sarin, R., Robbins, D.C.: Stuff I've seen: a system for personal information retrieval and re-use. In: Proceedings of the 26th Annual International ACM SIGIR Conference on Research and Development in Informaion Retrieval (SIGIR '03), pp. 72-79 (2003)

6. Fernando, Z.T., Marenzi, I., Nejdl, W., Kalyani, R.: Archiveweb: Collaboratively Extending and Exploring Web Archive Collections. In: Proceedings of 20th International Conference on Theory and Practice of Digital Libraries: Research and Advanced Technology for Digital Libraries (TPDL '16), pp. 107-118 (2016)

7. Gomes, D., Miranda, J., Costa, M.: A survey on web archiving initiatives. In: Proceedings of the 15th International Conference on Theory and Practice of Digital Libraries: Research and Advanced Technology for Digital Libraries (TPDL '11), pp. 1045-1050 (2011)

8. Jackson, A., Lin, J., Milligan, I., Ruest, N.: Desiderata for Exploratory Search Interfaces to Web Archives in Support of Scholarly Activities. In: Proceedings of the 16th Joint Conference on Digital Libraries, JCDL '16, pp. 103$106(2016)$

9. Lieser, W.: Digital Art (Art Pocket). H.F.Ullmann Publishing $\mathrm{GmbH}$ (2009)

10. Lin, J., Gholami, M., Rao, J.: Infrastructure for supporting exploration and discovery in web archives. In: Proceedings of the 23rd International Conference on World Wide Web (WWW'14), pp. 851-856 (2014)

11. Marenzi, I.: Multiliteracies and e-learning2.0. In: G. Blell, R. Kupetz (eds.) Foreign Language Pedagogy, Content and Learner Oriented, vol. 28. Peter Lang (2014)

12. Marenzi, I., Nejdl, W.: I search therefore I learn - Active and collaborative learning in language teaching: Two case studies, pp. 103-125. Collaborative Learning 2.0: Open Educational Resources. IGI Global (2012)

13. Marenzi, I., Zerr, S.: Multiliteracies and active learning in CLIL - the development of LearnWeb2.0. In: IEEE Transactions on Learning Technologies (TLT) (2012)

14. Odijk, D., Gârbacea, C., Schoegje, T., Hollink, L., de Boer, V., Ribbens, K., van Ossenbruggen, J.: Supporting Exploration of Historical Perspectives across Collections. In: Proceedings of 19th International Conference on Theory and Practice of Digital Libraries (TPDL '15), pp. 238-251 (2015)

15. Padia, K., AlNoamany, Y., Weigle, M.C.: Visualizing Digital Collections at Archive-It. In: Proceedings of the 12th ACM/IEEE-CS Joint Conference on Digital Libraries (JCDL '12), pp. 15-18 (2012)

16. Ras, M., van Bussel, S.: Web archiving user survey. Technical report, National Library of the Netherlands (Koninklijke Bibliotheek) (2007)

17. Stalker, P.J.: Gaming In Art: A Case Study Of Two Examples Of The Artistic Appropriation Of Computer Games and The Mapping Of Historical Trajectories Of 'Art Games' Versus Mainstream Computer Games. University of Witwatersrand (2005)

18. Weikum, G., Ntarmos, N., Spaniol, M., Triantafillou, P., Benczúr, A., Kirkpatrick, S., Rigaux, P., Williamson, M.: Longitudinal Analytics on Web Archive Data: It's About Time! In: Proceedings of the $5^{t h}$ biennial Conference on Innovative Data Systems Research (CIDR), Asilomar, CA, USA, January 9-12, pp. 199-202 (2011)

19. Winters, J.: Tackling complexity in humanities big data: From parliamentary proceedings to the archived web. In Turo Hiltunen, Joe McVeigh and Tanja Säily (eds.), Big and Rich Data in English Corpus Linguistics: Methods and Explorations. Studies in Variation, Contacts and
Change in English. Helsinki: VARIENG. (Forthcoming 2017) 